\title{
Measurement of Branching Fractions and Spectral Functions in $\tau$ Decays
}

\author{
Michel Davier ${ }^{a}$

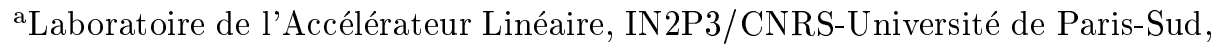 \\ BP34, 91898 Orsay, France
}

Full LEP-I data collected by the ALEPH detector during 1991-1995 running are analyzed in order to measure the $\tau$ decay branching fractions and the hadronic spectral functions. The analysis follows the global method used in the published study based on 1991-1993 data, with several improvements, especially concerning the treatment of photons and $\pi^{0}$ 's. Extensive systematic studies are performed, in order to match the large statistics of the data sample corresponding to 327148 measured and identified $\tau$ decays. Preliminary values for the branching fractions are obtained for the 2 leptonic channels and 11 hadronic channels defined by their respective numbers of charged particles and $\pi^{0}$ 's. Using previously published ALEPH results on final states with charged and neutral kaons, corrections are applied so that branching ratios for exclusive final states without kaons are derived. Some physics implications of the results are given, in particular concerning universality in the leptonic charged weak current, isospin invariance in $a_{1}$ decays, and the separation of vector and axial-vector components of the total hadronic rate. Spectral functions are obtained for the main exclusive channels and the total inclusive rate, with separation of the vector and axial-vector states.

\section{Introduction}

A complete and final analysis of $\tau$ decays is presented using a global method. All data recorded at LEP-I with the ALEPH detector are used, thus providing an update of those previous results which were based on partial data sets. The increase in statistics - the full sample corresponds to about 2.5 times the luminosity used in the last published global analysis [1,2] — not only allows for a reduction of the dominant statistical error but, more importantly, provides a way to better study and correct systematic biases. Several improvements of the method have been introduced in order to achieve a better control over the most relevant systematic uncertainties: simulation-independent measurement of the $\tau \tau$ selection efficiency, improved photon identification especially at low energy where the separation between photons from $\tau$ decays and fake photons from fluctuations in hadronic or electromagnetic showers is delicate, a new method to correct the Monte Carlo simulation for the rate of fake photons, and stricter criteria for channels with low branching fractions. For consistency and in order to maximally profit from the improved analysis all data sets recorded from 1991 to 1995 have been reprocessed. The results presented in this paper thus supersede those already published in Ref. [3,1,2]. Only the measurements on final states containing kaons, which were already based on the full statistics, remain unchanged [6-9].

\section{Experimental method}

\subsection{The data and simulated samples}

A detailed description of the ALEPH detector can be found elsewhere $[10,11]$.

Tau-pair events are simulated by means of a Monte Carlo program which includes initial state radiation computed up to order $\alpha^{2}$ and exponentiated, and final state radiative corrections to order $\alpha[12,13]$. The simulation of the subsequent $\tau$ decays also includes single photon radiation for the decays with up to three hadrons in the final state.

The data used in this analysis have been recorded at LEP I in 1991-1995, for a total number of detected $\tau$ decays of $3.3 \cdot 10^{5}$. 


\subsection{Selection of $\tau \tau$ events}

The principal characteristics of $\tau \tau$ events are low multiplicity, back-to-back topology and missing energy. Each event is divided into two hemispheres by an energy flow algorithm [11] which calculates all the visible energy avoiding doublecounting between the TPC and the calorimeter information. Cuts on the kinematic variables of the two hemispheres are used to veto Bhabha, muon-pair, hadronic $Z$ and $\gamma \gamma$-induced events $[14,1,2]$.

We use the 'break-mix' method introduced for the determination of the $\tau \tau$ cross section [14] to measure the efficiency of all the selection cuts. For every cut, one hemisphere of the event is chosen judiciously so that it is unbiased with respect to the cut under study and free of non- $\tau$ backgrounds. This procedure selects the opposite hemisphere as an unbiased $\tau$ decay which is then stored away. Pairs of selected hemispheres are combined to construct a $\tau \tau$ event sample built completely from data. This sample is used to measure the efficiency of the given cut.

The measured efficiencies are found to be very close to those obtained by the simulation, deviations being at most at the few per mille level. This situation stems from the facts that the $\tau$ decay dynamics is - apart from small branching ratio channels - very well known, the selection efficiencies are large and the simulation of the detector is adequate. The overall selection efficiency of $\tau \tau$ events is $78.8 \%$. This value increases to $91.9 \%$ when the $\tau \tau$ angular distribution is restricted to the detector polar acceptance, giving a better indication for the efficiency of the cuts designed to exclude non- $\tau \tau$ backgrounds. In addition, when expressed relatively to each $\tau$ decay, the selection efficiencies are weakly dependent on the final state, with a total relative span of only $10 \%$ for the 13 considered decay topologies.

\subsection{Estimation of non- $\tau \tau$ backgrounds}

A new method - already implemented for the measurement of the $\tau$ polarization [15] — has been developed to directly measure in the final data samples the contributions from the major non$\tau$ backgrounds. The procedure does not require an absolute normalization from the Monte Carlo simulation of these channels, but only a qualitative description of the distribution of the discriminating variables. The basic idea is to apply cuts on the data in order to reduce as much as possible the $\tau \tau$ population while keeping a high efficiency for the background source under study, i.e. the reverse of what is done in the $\tau \tau$ selection. The non- $\tau$ backgrounds in each channel are listed in Table 1. They amount to a total fraction of $(1.23 \pm 0.04) \%$ in the full data sample.

\subsection{Charged particle identification}

Charged particle identification is achieved with a likelihood method incorporating the information from the relevant detectors. In this way, each charged particle is assigned a set of probabilities from which a particle type is chosen. No attempt is made in this analysis to separate kaons from pions in the hadron sample since final states containing kaons have been previously studied [68]. The performance of the particle identification has been studied in detail using control samples of Bhabha events, $\mu \mu$ pairs, $\gamma \gamma$-induced lepton pairs and hadrons from $\pi^{0}$-tagged $\tau$ decays over the full angular and momentum range [1].

\subsection{Photon identification}

The high collimation of $\tau$ decays at LEP energies quite often makes photon reconstruction difficult, since these photons are close to one another or close to the showers generated by charged hadrons. Of particular relevance is the rejection of fake photons which may occur because of hadronic interactions, fluctuations of electromagnetic showers, or the overlapping of several showers. These problems reach a tolerable level thanks to the fine granularity of ECAL, in both transverse and longitudinal directions, but nevertheless they require the development of proper and reliable methods in order to correctly identify photon candidates.

A likelihood method is used for discriminating between genuine and fake photons. For every cluster above a threshold of $300 \mathrm{MeV}$, an estimator $P_{\gamma}$ is defined, $P_{\gamma}=0,1$ corresponding to fake and real photons,respectively. It is constructed using probability densities of discriminating variables obtained by simulation, but 
corrected through detailed comparisons between data and Monte Carlo. Major improvements were introduced at this stage in the analysis compared to the previous one [2], mainly in the choice of variables and in the use of energy-dependent reference distributions. Better photon energy calibration is achieved compared to the previous analysis. Photon conversions are also reconstructed.

\section{6. $\pi^{0}$ reconstruction}

Three different kinds of $\pi^{0}$ 's are reconstructed: resolved $\pi^{0}$ from two-photon pairing, unresolved $\pi^{0}$ from merged clusters, and residual $\pi^{0}$ from the remaining single photons after removing radiative, bremsstrahlung and fake photons with a likelihood method.

After the pairing and the cluster moment analysis, all the remaining photons inside a cone of $30^{\circ}$ around the jet axis are considered. Radiative and bremsstrahlung photons are selected using a likelihood method and are kept separate in the $\tau$ decay classification discussed below. The identification of final state radiative photons in hadronic channels is a difficult task and and to a large extent the analysis relies on the description of radiation at the generator level in the Monte Carlo [16].

\subsection{Decay classification}

Each $\tau$ decay is classified topologically according to the number of hadronic charged tracks, their particle identification and the number of $\pi^{0}$ 's reconstructed. While for 1-prong and 5prong channels the exact multiplicity is required, the track number in 3-prong channels is allowed to be 2,3 or 4 , in order to reduce systematic effects due to tracking and secondary interactions. The definition of the leptonic channels requires an identified electron or muon with any number of photons. Hemispheres rejected by specific cuts are put in a special class, labelled 14, which does not correspond to a nominal physical $\tau$ decay mode. The numbers of $\tau$ 's classified in each of the considered decay channels are listed in Table 1.

The KORAL07 generator [12] in the Monte Carlo simulation incorporates all the decay modes considered in this analysis, except for the $h 4 \pi^{0}$
Table 1

Numbers of reconstructed and estimated non- $\tau$ background events in 1991-1993 and 1994-1995 data sets in the different considered topologies.

\begin{tabular}{l|rr|rr}
\hline \hline class & $91-93$ & & $94-95$ & \\
\hline$e$ & 22405 & $598 \pm 46$ & 33100 & $745 \pm 58$ \\
$\mu$ & 22235 & $409 \pm 45$ & 32145 & $380 \pm 40$ \\
$h$ & 15126 & $93 \pm 11$ & 22429 & $100 \pm 13$ \\
$h \pi^{0}$ & 32282 & $141 \pm 22$ & 49008 & $178 \pm 26$ \\
$h 2 \pi^{0}$ & 12907 & $44 \pm 9$ & 18317 & $81 \pm 16$ \\
$h 3 \pi^{0}$ & 2681 & $26 \pm 7$ & 3411 & $35 \pm 9$ \\
$h 4 \pi^{0}$ & 458 & $12 \pm 3$ & 499 & $19 \pm 5$ \\
$3 h$ & 11610 & $87 \pm 20$ & 17315 & $129 \pm 30$ \\
$3 h \pi^{0}$ & 6467 & $97 \pm 23$ & 9734 & $165 \pm 39$ \\
$3 h 2 \pi^{0}$ & 1091 & $27 \pm 7$ & 1460 & $36 \pm 10$ \\
$3 h 3 \pi^{0}$ & 124 & $13 \pm 4$ & 150 & $25 \pm 7$ \\
$5 h$ & 60 & $3 \pm 1$ & 105 & $7 \pm 2$ \\
$5 h \pi^{0}$ & 36 & $16 \pm 5$ & 59 & $21 \pm 6$ \\
14 & 4834 & $249 \pm 38$ & 7100 & $303 \pm 52$ \\
\hline sum & 132316 & $1815 \pm 86$ & 194832 & $2224 \pm 107$ \\
\hline \hline
\end{tabular}

decay channel for which a separate generation was done using a phase space model. The complete behaviour between the generated decays and their reconstructed counterparts using the decay classification is embodied in the efficiency matrix. This matrix $\varepsilon_{j i}$ gives the probability of a $\tau$ decay generated in class $j$ to be reconstructed in class $i$. Obtained initially using the simulated samples, it is corrected for effects where data and simulation can possibly differ, such as particle identification and photon identification. For the latter effect, a detailed procedure has been developed in order to correct the simulation of fake photons by comparison with data.

\section{Results}

\subsection{Determination of the branching ratios}

The branching ratios are determined using

$$
\begin{aligned}
n_{i}^{o b s}-n_{i}^{b k g} & =\sum_{j} \varepsilon_{j i} N_{j}^{\text {prod }} \\
B_{j} & =\frac{N_{j}^{\text {prod }}}{\sum_{j} N_{j}^{\text {prod }}}
\end{aligned}
$$


where $n_{i}^{o b s}$ is the observed events number in reconstructed class $i, n_{i}^{b k g}$ the non- $\tau$ background in reconstructed class $i, \varepsilon_{j i}$ the efficiency of a produced class $j$ event reconstructed as class $i$, and $N_{j}^{\text {prod }}$ the produced events number of class $j$. The class numbers $i, j$ run from 1 to 14 , the last one corresponding to the rejected $\tau$ candidates.

The analysis assumes a standard $\tau$ decay description. One could imagine unknown decay modes not included in the simulation, but since large detection efficiencies are achieved in the $\tau \tau$ selection which is therefore robust, it would be difficult for these decays to pass unnoticed. An independent measurement of the branching ratio for undetected decay modes, using a direct search with a one-sided $\tau$ tag, was done in ALEPH [17], limiting this branching ratio to less than $0.11 \%$ at $95 \% \mathrm{CL}$. This result justifies the assumption that the sum of the branching ratios for visible $\tau$ decays is equal to one.

The branching ratios are obtained and listed in Table 2. The most important systematic uncertainties originate from photon identification and $\pi^{0}$ reconstruction, and secondary interactions.

\subsection{From reconstructed classes to exclu- sive modes}

The 13 classes corresponding to major $\tau$ decay modes still contain the contributions from final states involving kaons. The latter are coming from Cabibbo-suppressed $\tau$ decays or modes with a $K \bar{K}$ pair, both characterized by small branching ratios compared to the nonstrange modes without kaons. Complete analyses of $\tau$ decays involving neutral or charged kaons have been performed by ALEPH on the full LEP I data [6-8]. They are summarized and measurements with $K_{S}^{0}$ and $K_{L}^{0}$ are combined in Ref. [9].

The $\tau$ decays involving $\eta$ or $\omega$ mesons also require special attention in this analysis because single photons in their electromagnetic decay modes are treated as $\pi^{0}$ candidates. Corrections are applied, based on specific measurements by ALEPH [18] and CLEO [20] of $\tau$ decay modes containing those mesons. This bookkeeping takes into account all the major decay modes of the considered mesons [19], including the isospinviolating $\omega \rightarrow \pi^{+} \pi^{-}$decay mode. Some much smaller contributions $3 \pi(\eta, \omega)$ have been identified and measured by CLEO [21]. Even though the corrections from these channels are very small they have been included for the sake of completeness. Finally, another very small correction has been applied to take into account the $a_{1}$ radiative decay into $\pi \gamma[22]$.

\subsection{Overall consistency test}

Rejected $\tau$ hemispheres because of charged particle identification cuts $(2 \mathrm{GeV}$ minimum momentum and ECAL crack veto for some 1-prong modes, strict definition of higher multiplicity channels) are placed in class 14 . As already emphasized, this sample does not correspond to a nominal $\tau$ decay mode and should be explained by all other measured fractions in the other classes and the efficiency matrix. Thus the determination of a hypothetical signal in this class is a measure of the level of consistency achieved in the analysis.

For this determination the efficiency of the possible signal in class 14 is taken to be $100 \%$. The results, already shown in Table 2 separately for the 1991-1993 and 1994-1995 data sets, are consistent and are combined to give $B_{14}=(0.066 \pm$ $0.042 \%$. This value is consistent with zero and provides a good check of the overall procedure at the $0.1 \%$ level for branching ratios. It coincides, approximately and accidentally, with the limit achieved of $0.11 \%$ at $95 \%$ CL in a direct search for 'invisible' decays not selected in the 13-channel classification. In the following it is assumed that all $\tau$ decay modes have been properly considered at the $0.1 \%$ precision level and no physics contribution beyond standard $\tau$ decays is further allowed. Thus the quantity $B_{14}$ is now constrained to be zero.

It can be further noticed that this analysis provides a branching ratio in the $3 \pi 3 \pi^{0}$ class which is consistent with zero (see Table 2). The result is therefore given as an upper limit at $95 \%$ $\mathrm{CL}, B_{3 \pi 3 \pi^{0}}<4.910^{-4}$, consistent with the measurement made by CLEO [23] yielding $B_{3 \pi 3 \pi^{0}}=$ $(2.2 \pm 0.5) 10^{-4}$. The final state is dominated by $\eta$ and $\omega$ resonances [23] and using other channels allows one to obtain a lower limit for this branching ratio, $(2.6 \pm 0.4) 10^{-4}$. In the following a value of 
Table 2

Branching ratios (\%) for the reconstructed topologies (quasi-exclusive modes) from 1991-1993 and 19941995 data sets; the first error is statistical and the second is systematic.

\begin{tabular}{|l|c|c|}
\hline \hline class & $91-93$ & $94-95$ \\
\hline$\mu$ & $17.859 \pm 0.112 \pm 0.058$ & $17.799 \pm 0.093 \pm 0.045$ \\
$\mu$ & $17.356 \pm 0.107 \pm 0.055$ & $17.273 \pm 0.087 \pm 0.039$ \\
$h$ & $12.238 \pm 0.105 \pm 0.104$ & $12.058 \pm 0.088 \pm 0.083$ \\
$h \pi^{0}$ & $26.132 \pm 0.150 \pm 0.104$ & $26.325 \pm 0.123 \pm 0.090$ \\
$h 2 \pi^{0}$ & $9.680 \pm 0.139 \pm 0.124$ & $9.663 \pm 0.107 \pm 0.105$ \\
$h 3 \pi^{0}$ & $1.128 \pm 0.110 \pm 0.086$ & $1.229 \pm 0.089 \pm 0.068$ \\
$h 4 \pi^{0}$ & $0.227 \pm 0.056 \pm 0.047$ & $0.163 \pm 0.050 \pm 0.040$ \\
$3 h$ & $9.931 \pm 0.097 \pm 0.072$ & $9.769 \pm 0.080 \pm 0.059$ \\
$3 h \pi^{0}$ & $4.777 \pm 0.093 \pm 0.074$ & $4.965 \pm 0.077 \pm 0.066$ \\
$3 h 2 \pi^{0}$ & $0.517 \pm 0.063 \pm 0.050$ & $0.551 \pm 0.050 \pm 0.038$ \\
$3 h 3 \pi^{0}$ & $0.016 \pm 0.029 \pm 0.020$ & $-0.021 \pm 0.023 \pm 0.019$ \\
$5 h$ & $0.098 \pm 0.014 \pm 0.006$ & $0.098 \pm 0.011 \pm 0.004$ \\
$5 h \pi^{0}$ & $0.022 \pm 0.010 \pm 0.009$ & $0.028 \pm 0.008 \pm 0.007$ \\
14 & $0.017 \pm 0.043 \pm 0.042$ & $0.099 \pm 0.035 \pm 0.037$ \\
\hline \hline
\end{tabular}

$(3 \pm 1) 10^{-4}$ is used as input for this channel and the global analysis is performed in terms of the remaining 12 defined channels which are refitted.

\subsection{Final combined results}

The two sets of results are consistent and are finally combined in Table 3 .

The branching ratios obtained for the different channels are correlated with each other. On one hand the statistical fluctuations in the data and the Monte Carlo samples are driven by the multinomial distribution of the corresponding events, producing well-understood correlations. On the other hand the systematic effects also induce important and nontrivial correlations between the different channels. All the systematic studies were done keeping track of the correlated variations in the final branching ratio results, thus allowing a proper propagation of errors.

The present results are consistent with those of the previously published ALEPH analysis [1, 2]. The leptonic branching ratios also agree within errors with the results of an independent ALEPH analysis which does not rely on a global method [24].

\section{Discussion of the results}

\subsection{Comparison with other experiments}

Figs. 1, 2, 3, 4 show that the results of this analysis are in good agreement with those from other most precise experiments. In all these cases, ALEPH achieves the best precision.

\subsection{Universality in the leptonic charged current}

\subsection{1. $\mu$-e universality from the leptonic branching ratios}

In the standard V-A theory with leptonic coupling $g_{l}$ at the $W l \bar{\nu}_{l}$ vertex, the $\tau$ leptonic partial width can be computed, including radiative corrections [25] and safely neglecting neutrino masses:

$\Gamma\left(\tau \rightarrow \nu_{\tau} l \bar{\nu}_{l}(\gamma)\right)=\frac{G_{\tau} G_{l} m_{\tau}^{5}}{192 \pi^{3}} f\left(\frac{m_{l}^{2}}{m_{\tau}^{2}}\right) \delta_{W}^{\tau} \delta_{\gamma}^{\tau}$,

where

$$
\begin{aligned}
G_{l} & =\frac{g_{l}^{2}}{4 \sqrt{2} M_{W}^{2}} \\
\delta_{W}^{\tau} & =1+\frac{3}{5} \frac{m_{\tau}^{2}}{M_{W}^{2}} \\
\delta_{\gamma}^{\tau} & =1+\frac{\alpha\left(m_{\tau}\right)}{2 \pi}\left(\frac{25}{4}-\pi^{2}\right)
\end{aligned}
$$


Table 3

Combined results for the exclusive branching ratios (B) for modes without kaons. The contributions from channels with $\eta$ and $\omega$ are given separately, the latter only for the electromagnetic $\omega$ decays. All results are from this analysis, unless explicitly stated. The values labelled ${ }^{*}$ and ${ }^{* *}, * * *$ are taken from ALEPH [18] and CLEO [21,20], respectively.

\begin{tabular}{lr}
\hline \hline mode & $\mathrm{B} \pm \sigma_{\text {Stat }} \pm \sigma_{\text {Syst }}[\%]$ \\
\hline$e$ & $17.837 \pm 0.072 \pm 0.036$ \\
$\mu$ & $17.319 \pm 0.070 \pm 0.032$ \\
$\pi^{-}$ & $10.828 \pm 0.070 \pm 0.078$ \\
$\pi^{-} \pi^{0}$ & $25.471 \pm 0.097 \pm 0.085$ \\
$\pi^{-} 2 \pi^{0}$ & $9.239 \pm 0.086 \pm 0.090$ \\
$\pi^{-} 3 \pi^{0}$ & $0.977 \pm 0.069 \pm 0.058$ \\
$\pi^{-} 4 \pi^{0}$ & $0.112 \pm 0.037 \pm 0.035$ \\
$\pi^{-} \pi^{-} \pi^{+}$ & $9.041 \pm 0.060 \pm 0.076$ \\
$\pi^{-} \pi^{-} \pi^{+} \pi^{0}$ & $4.590 \pm 0.057 \pm 0.064$ \\
$\pi^{-} \pi^{-} \pi^{+} 2 \pi^{0}$ & $0.392 \pm 0.030 \pm 0.035$ \\
$\pi^{-} \pi^{-} \pi^{+} 3 \pi^{0}($ estim. $)$ & $0.013 \pm 0.000 \pm 0.010$ \\
$3 \pi^{-} 2 \pi^{+}$ & $0.072 \pm 0.009 \pm 0.012$ \\
$3 \pi^{-} 2 \pi^{+} \pi^{0}$ & $0.014 \pm 0.007 \pm 0.006$ \\
$\pi^{-} \pi^{0} \eta^{*}$ & $0.180 \pm 0.040 \pm 0.020$ \\
$\pi^{-} 2 \pi^{0} \eta^{* *}$ & $0.015 \pm 0.004 \pm 0.003$ \\
$\pi^{-} \pi^{-} \pi^{+} \eta^{* *}$ & $0.024 \pm 0.003 \pm 0.004$ \\
$a_{1}^{-}\left(\rightarrow \pi^{-} \gamma\right)(\mathrm{estim})$. & $0.040 \pm 0.000 \pm 0.020$ \\
$\pi^{-} \omega\left(\rightarrow \pi^{0} \gamma, \pi^{+} \pi^{-}\right)^{*}$ & $0.253 \pm 0.005 \pm 0.017$ \\
$\pi^{-} \pi^{0} \omega\left(\rightarrow \pi^{0} \gamma, \pi^{+} \pi^{-}\right)^{*, * * *}$ & $0.048 \pm 0.006 \pm 0.007$ \\
$\pi^{-} 2 \pi^{0} \omega\left(\rightarrow \pi^{0} \gamma, \pi^{+} \pi^{-}\right)^{* *}$ & $0.002 \pm 0.001 \pm 0.001$ \\
$\pi^{-} \pi^{-} \pi^{+} \omega\left(\rightarrow \pi^{0} \gamma, \pi^{+} \pi^{-}\right)^{* *}$ & $0.001 \pm 0.001 \pm 0.001$ \\
\hline \hline
\end{tabular}

$f(x)=1-8 x+8 x^{3}-x^{4}-12 x^{2} \ln x$

Numerically, the $\mathrm{W}$ propagator correction and the radiative corrections are small: $\delta_{W}^{\tau}=1+$ $2.9 \cdot 10^{-4}$ and $\delta_{\gamma}^{\tau}=1+43.2 \cdot 10^{-4}$.

Taking the ratio of the two leptonic branching fractions, a direct test of $\mu$-e universality is obtained. The measured ratio

$\frac{B_{\mu}}{B_{e}}=0.9709 \pm 0.0060 \pm 0.0029$

agrees with the expectation which is equal to 0.97257 when universality holds. Alternatively the measurements yield the ratio of couplings

$\frac{g_{\mu}}{g_{e}}=0.9991 \pm 0.0033$

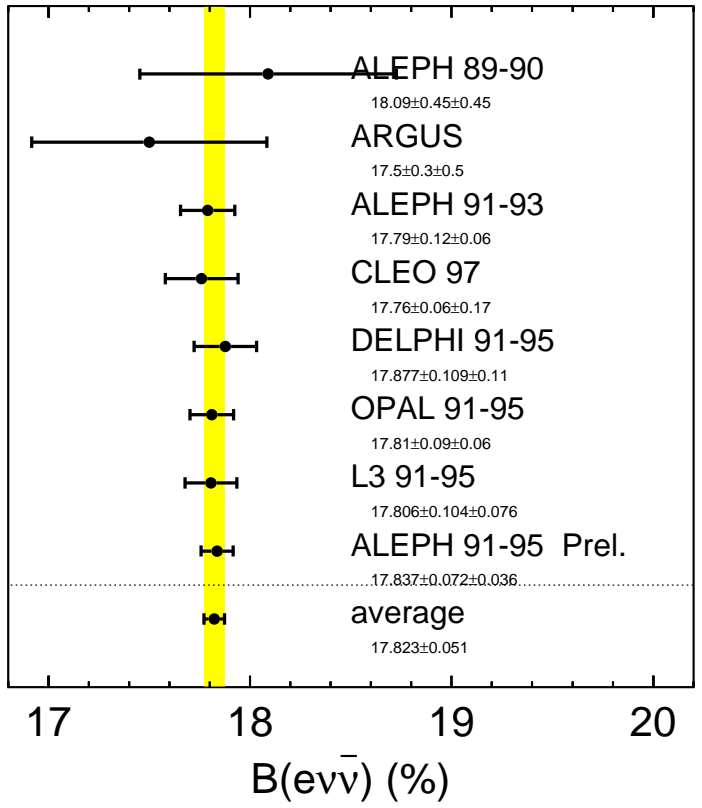

Figure 1. Comparison of ALEPH measurement with published precise results from other experiments for $\tau \rightarrow e \nu \bar{\nu}$.

which is consistent with one.

This result is in agreement with the best test of $\mu$-e universality of the $\mathrm{W}$ couplings obtained in the comparison of the rates for $\pi \rightarrow \mu \bar{\nu}_{\mu}$ and $\pi \rightarrow e \bar{\nu}_{e}$ decays, where the results of the two most accurate experiments $[26,27]$ can be averaged to yield $\frac{g_{\mu}}{g_{e}}=1.0012 \pm 0.0016$. The results have comparable precision, but it should be pointed out that they are in fact complementary. The $\tau$ result given here probes the coupling to a transverse $W$ (helicity \pm 1 ) while the $\pi$ decays measure the coupling to a longitudinal W (helicity 0 ). It is indeed conceivable that either approach could be sensitive to different nonstandard corrections to universality.

\subsubsection{Tests of $\tau$ - $\mu$ and $\tau$-e universality}

Comparing the rates for $\Gamma\left(\tau \rightarrow \nu_{\tau} e \bar{\nu}_{e}(\gamma)\right)$, $\Gamma\left(\tau \rightarrow \nu_{\tau} \mu \bar{\nu}_{\mu}(\gamma)\right)$ and $\Gamma\left(\mu \rightarrow \nu_{\mu} e \bar{\nu}_{e}(\gamma)\right)$ provides direct tests of the universality of $\tau$ - $\mu$-e couplings. Taking the relevant ratios with calculated radia- 


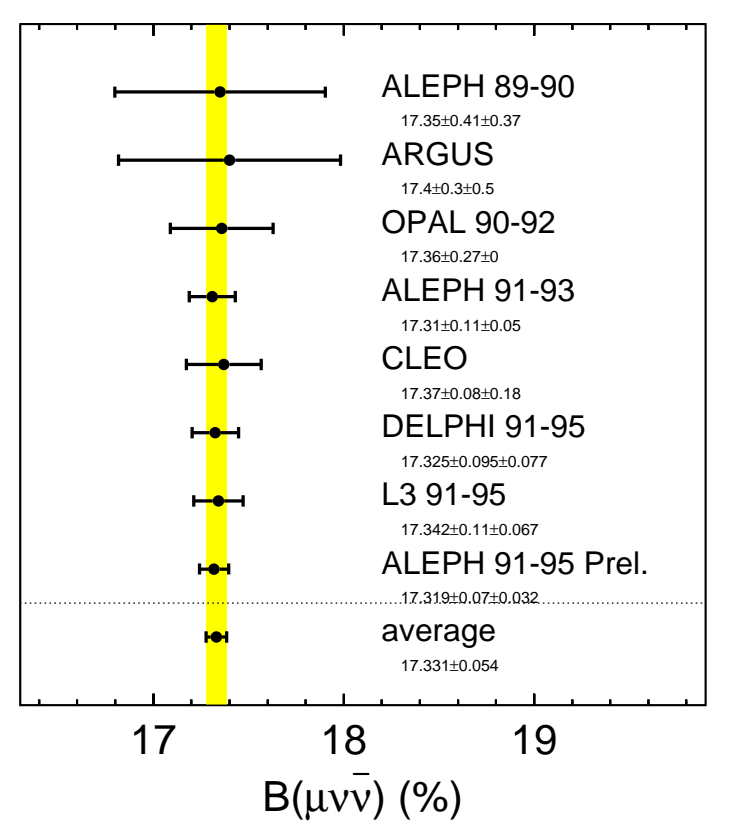

Figure 2. Comparison of ALEPH measurement with published precise results from other experiments for $\tau \rightarrow \mu \nu \bar{\nu}$.

tive corrections, one obtains

$$
\begin{aligned}
\left(\frac{g_{\tau}}{g_{\mu}}\right)^{2} & =\frac{\tau_{\mu}}{\tau_{\tau}}\left(\frac{m_{\mu}}{m_{\tau}}\right)^{5} B_{e} \frac{f\left(\frac{m_{e}^{2}}{m_{\mu}^{2}}\right)}{f\left(\frac{m_{e}^{2}}{m_{\tau}^{2}}\right)} \Delta_{W} \Delta_{\gamma} \\
\left(\frac{g_{\tau}}{g_{e}}\right)^{2} & =\frac{\tau_{\mu}}{\tau_{\tau}}\left(\frac{m_{\mu}}{m_{\tau}}\right)^{5} B_{\mu} \frac{f\left(\frac{m_{e}^{2}}{m_{\mu}^{2}}\right)}{f\left(\frac{m_{\mu}^{2}}{m_{\tau}^{2}}\right)} \Delta_{W} \Delta_{\gamma}
\end{aligned}
$$

where $f\left(\frac{m_{e}^{2}}{m_{\mu}^{2}}\right)=0.9998, \Delta_{W}=\frac{\delta_{W}^{\mu}}{\delta_{W}^{\tau}}=1-2.9 \cdot 10^{-4}$, $\Delta_{\gamma}=\frac{\delta_{\gamma}^{\mu}}{\delta_{\gamma}^{\tau}}=1+8.5 \cdot 10^{-5}$, and $\tau_{l}$ is the lepton $l$ lifetime.

From the present measurements of $B_{e}, B_{\mu}$, the $\tau$ mass [19], $m_{\tau}=\left(1777.03_{-0.26}^{+0.30}\right) \mathrm{MeV}$ (dominated by the BES result [28]), the $\tau$ lifetime [19], $\tau_{\tau}=(290.6 \pm 1.1)$ fs and the other quantities from Ref. [19], universality can be tested:

$$
\frac{g_{\tau}}{g_{\mu}}=1.0009 \pm 0.0023 \pm 0.0019 \pm 0.0004
$$

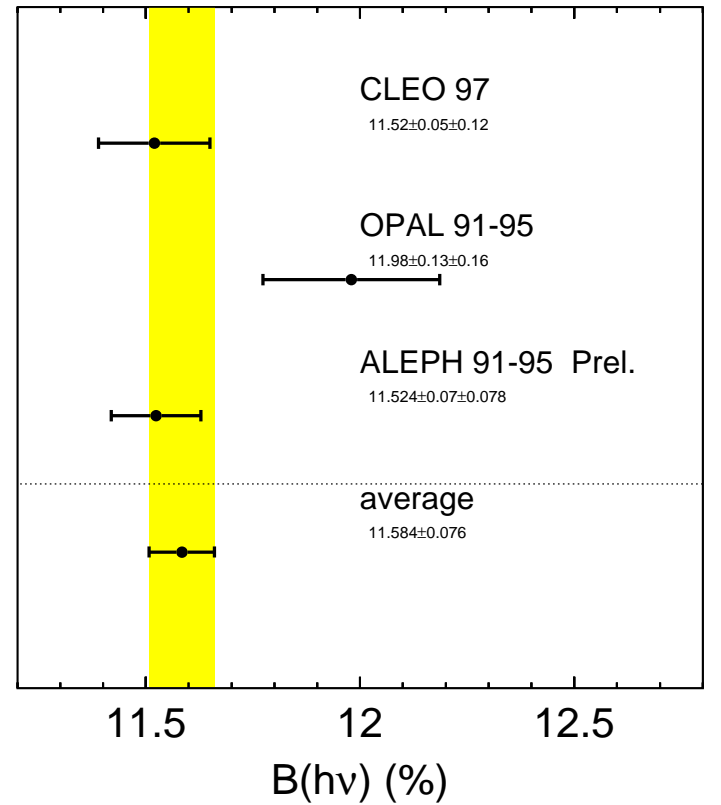

Figure 3. Comparison of ALEPH measurement with published precise results from other experiments for $\tau \rightarrow h \nu$ (sum of $\pi \nu$ and $K \nu$ ).

$\frac{g_{\tau}}{g_{e}}=1.0001 \pm 0.0022 \pm 0.0019 \pm 0.0004$,

where the errors are from the corresponding leptonic branching ratio and the $\tau$ lifetime and mass, respectively.

\subsection{3. $\tau-\mu$ universality from the pionic branching ratio}

The measurement of $B_{\pi}$ also permits an independent test of $\tau$ - $\mu$ universality through the relation

$$
\begin{aligned}
\left(\frac{g_{\tau}}{g_{\mu}}\right)^{2}= & \frac{B_{\pi}}{B_{\pi \rightarrow \mu \bar{\nu} \mu}} \frac{\tau_{\pi}}{\tau_{\tau}} \frac{2 m_{\pi} m_{\mu}^{2}}{m_{\tau}^{3}} \\
& \times\left(\frac{1-m_{\mu}^{2} / m_{\pi}^{2}}{1-m_{\pi}^{2} / m_{\tau}^{2}}\right)^{2} \delta_{\tau / \pi},
\end{aligned}
$$

where the radiative correction [29] amounts to $\delta_{\tau / \pi}=1.0016 \pm 0.0014$ Using the world-averaged values for the $\tau$ and $\pi$ ( $\tau_{\tau}$ and $\left.\tau_{\pi}\right)$ lifetimes, and 


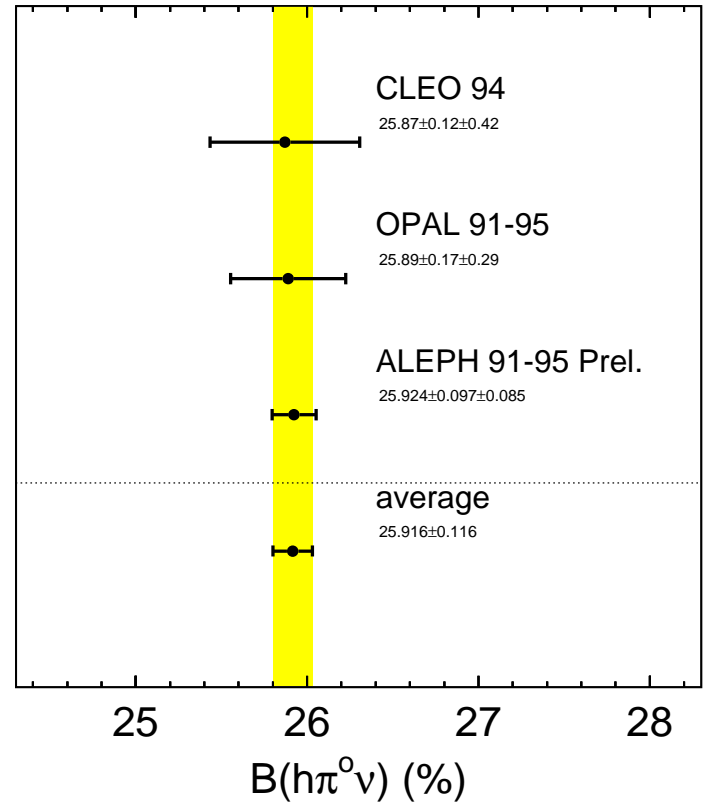

Figure 4. Comparison of ALEPH measurement with published precise results from other experiments for $\tau \rightarrow h \pi^{0} \nu$ (sum of $\pi \pi^{0} \nu$ and $K \pi^{0} \nu$ ).

the branching ratio for the decay $\pi \rightarrow \mu \nu$ [19], the present result for $B_{\pi}$, one obtains

$\frac{g_{\tau}}{g_{\mu}}=0.9962 \pm 0.0048 \pm 0.0019 \pm 0.0002$,

comparing the measured value $\left(B_{\pi}=10.823 \pm\right.$ $0.104) \%$ to the expected one assuming universality $(10.910 \pm 0.042) \%$. The quoted errors in Eq. 12 are from the pion mode branching ratio and the $\tau$ lifetime and mass, respectively.

The two determinations of $\frac{g_{\tau}}{g_{\mu}}$ obtained from $B_{e}$ and $B_{\pi}$ are consistent with each other and can be combined to yield

$\frac{g_{\tau}}{g_{\mu}}=1.0000 \pm 0.0021 \pm 0.0019 \pm 0.0004$,

where the errors are from the electron and pion branching ratio and the $\tau$ lifetime and mass, respectively. Universality of the $\tau$ and $\mu$ chargedcurrent couplings holds at the $0.29 \%$ level with about equal contributions from the present deter-

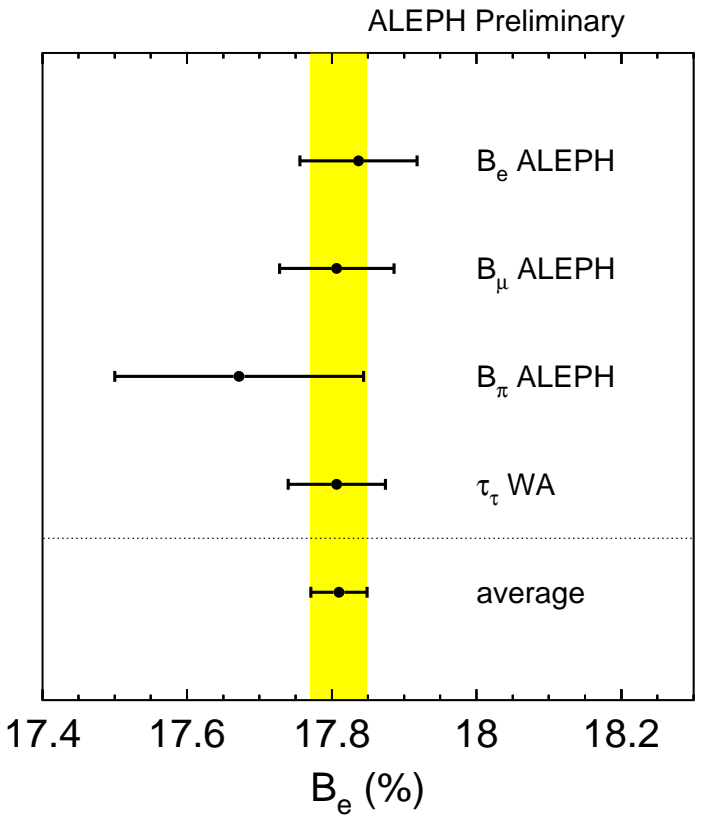

Figure 5. The measured value for $B_{e}$ compared to predictions from other measurements assuming leptonic universality. The vertical band gives the average of all determinations.

mination of $B_{e}$ and $B_{\pi}$, and the world-averaged value for the $\tau$ lifetime.

The consistency of the present branching ratio measurements with leptonic universality is displayed in Fig. 5 where the result for $B_{e}$ is compared to computed values of $B_{e}$ using as input $B_{\mu}$ (assuming $e-\mu$ universality), $\tau_{\tau}$ and $\tau_{\mu}(\mu-\tau$ universality), and $B_{\pi}$ and $\tau_{\pi}$ ( $\mu-\tau$ universality). All values are consistent and yield the average

$B_{e}^{\text {universality }}=(17.810 \pm 0.039) \%$.

4.2.4. $a_{1}$ decays to $3 \pi$ and $\pi 2 \pi^{0}$

With the level of precision reached it is interesting to compare the rates in the $3 \pi$ and $\pi 2 \pi^{0}$ channels which are completely dominated by the $a_{1}$ resonance. The dominant $\rho \pi$ intermediate state leads to equal rates, but a small isospin-breaking effect is expected from different charged and neutral $\pi$ masses, slightly favouring the $\pi 2 \pi^{0}$ chan- 
nel.

A recent CLEO partial-wave analysis of the $\pi 2 \pi^{0}$ final state [30] has shown that the situation is in fact much more complicated with many intermediate states, in particular involving isoscalars, amounting to about $20 \%$ of the total rate and producing strong interference effects. A good description of the $a_{1}$ decays was achieved in the CLEO study, which can be applied to the $3 \pi$ final state, predicting [30] a ratio of the rates $3 \pi / \pi 2 \pi^{0}$ equal to 0.985 . This value, which includes known isospin-breaking from the pion masses, turns out to be in good agreement with the measured value from this analysis which shows the expected trend

$$
\frac{B_{3 \pi}}{B_{\pi 2 \pi^{0}}}=0.979 \pm 0.018 \text {. }
$$

\subsubsection{The $\pi \pi^{0}$ branching ratio in the con- text of $a_{\mu}^{\text {had }}$}

The $\pi \pi^{0}$ final state is dominated by the $\rho$ resonance. Its mass distribution - or better the corresponding spectral function - is a basic ingredient of vacuum polarization calculations, such as that used for computing the hadronic contribution to the anomalous magnetic moment of the muon $a_{\mu}^{\text {had }}$. In this case the $\rho$ contribution is dominant $(71 \%)$ and therefore controls the final precision of the result. It was proposed in Ref. [31] to use the spectral functions obtained from the measurement of hadronic $\tau$ decays in order to improve the precision of the prediction for $a_{\mu}^{h a d}$. The calculation was later improved with the help of QCD constraints for energies above the $\tau$ mass [32] and even below [33].

The normalization of the spectral function is provided by the branching fraction $B_{\pi \pi^{0}}$. The present world average is completely dominated by the published ALEPH result [2]. The new result given here is larger by $0.68 \%$, thus one can expect a slightly larger contribution to $a_{\mu}^{\text {had }}$.

A new evaluation [34] was available, using the preliminary spectral functions from the present analysis, the published CLEO results [35] and new results from $e^{+} e^{-}$annihilation from CMD2 [36]. Revision of the CMD-2 results [37] prompted a re-evaluation [38], which revealed a disagreement between the $\tau$ and $e^{+} e^{-}$spectral functions. Whereas the $\tau$ estimate leads to a prediction on the muon magnetic moment in agreement with the latest most precise measurement from the BNL experiment E-821 [39], the predicted value using only $e^{+} e^{-}$data lies 2.4 standard deviations below the measurement. In view of this situation, it is important to check all the ingredients, in particular the determination of the branching ratio $B_{\pi \pi^{0}}$.

As most of the systematic uncertainty in $B_{\pi \pi^{0}}$ comes from $\gamma / \pi^{0}$ reconstruction, it is interesting to cross check the results in the 'adjacent' hadronic modes, i.e. the $\pi$ and $\pi 2 \pi^{0}$ channels. This is possible if universality in the weak charged current is assumed, leading to an absolute prediction of $B_{\pi}$ using as input the $\tau$ lifetime (see Section 4.2.2), and by computing $B_{\pi 2 \pi^{0}}$ from the measurement of $B_{3 \pi}$ which is essentially uncorrelated with $B_{\pi \pi^{0}}$ (see Section 4.2.4). The two comparisons, $B_{\pi}-B_{\pi}^{u n i}=(-0.08 \pm 0.11) \%$, $B_{\pi 2 \pi^{0}}-B_{\pi 2 \pi^{0}}^{i s o}=(0.06 \pm 0.16) \%$, do not point to any systematic bias in the determination of $B_{\pi \pi^{0}}$ within the quoted uncertainty.

\subsubsection{Separation of vector and axial- vector contributions}

From the complete analysis of the $\tau$ branching ratios presented in this paper, it is possible to determine the nonstrange vector $(\mathrm{V})$ and axialvector (A) contributions to the total $\tau$ hadronic width, conveniently expressed in terms of their ratios to the leptonic width, called $R_{\tau, V}$ and $R_{\tau, A}$, respectively. The determination of the strange counterpart $R_{\tau, S}$ is already published [9].

The ratio $R_{\tau}$ for the total hadronic width is calculated from the difference of the ratio of the total hadronic width and electronic branching ratio,

$$
\begin{aligned}
R_{\tau} & =\frac{1-B_{e}-B_{\mu}}{B_{e}}=\frac{1}{B_{e}}-1.97257 \\
& =3.642 \pm 0.012
\end{aligned}
$$

taking for $B\left(\tau^{-} \rightarrow e^{-} \bar{\nu}_{e} \nu_{\tau}\right)$ the value obtained in Section 4.2 assuming universality in the leptonic weak current. Using the ALEPH measurement of the strange width ratio [9], very slightly modified to take into account the channel $K^{*-} \eta$ measured 
by CLEO [40]

$R_{\tau, S}=0.1603 \pm 0.0064$

the following result is obtained for the nonstrange component

$R_{\tau, V+A}=3.482 \pm 0.014$.

Separation of $\mathrm{V}$ and $\mathrm{A}$ components in hadronic final states with only pions is straightforward. Isospin invariance relates the spin-parity of such systems to their number of pions: G-parity $=1$ (even number) corresponds to vector states, while $\mathrm{G}=-1$ (odd number) tags axial-vector states. This property places a strong requirement on the efficiency of $\pi^{0}$ reconstruction, a constraint that was strongly integrated in this analysis.

Modes with a $K \bar{K}$ pair are not in general eigenstates of G-parity and contribute to both $\mathrm{V}$ and A channels. While the decay to $K^{-} K^{0}$ is pure vector, the situation in the $K \bar{K} \pi$ mode is not clear and a conservative axial-vector fraction of $0.75 \pm 0.25$ is assumed. For the decays into $K \bar{K} \pi \pi$ no information is available in this respect and a fraction $0.5 \pm 0.5$ is taken.

The total nonstrange vector and axial-vector contributions obtained in this analysis are:

$R_{\tau, V}=1.787 \pm 0.011 \pm 0.007$,

$R_{\tau, A}=1.695 \pm 0.011 \pm 0.007$,

where the second errors reflect the uncertainties in the $V / A$ separation in the channels with $K \bar{K}$ pairs. Taking care of the correlations between the respective uncertainties, one obtains the difference between the vector and axial-vector components, which is physically related to the amount of nonperturbative QCD contributions in the nonstrange hadronic $\tau$ decay width:

$R_{\tau, V-A}=0.092 \pm 0.018 \pm 0.014$

where again the second error has the same meaning as in Eqs. (19) and (20). The ratio

$\frac{R_{\tau, V-A}}{R_{\tau, V+A}}=0.026 \pm 0.007$

is a measure of the relative importance of nonperturbative QCD contributions.

\subsection{Summary of branching fractions}

The results presented here are combined with previously published ALEPH results on final states with kaons in Table 4.

\section{Spectral functions}

The hadronic mass spectra in the different exclusive channels $i$ are corrected by the appropriate kinematic factor and normalized by $B_{i} / B_{e}$ in 140 mass bins. The corrected spectra are unfolded from detector effects by a regularized inversion of the $140 \times 140$ detector response matrix [41]. Studies are performed using the full analysis for every source of systematic uncertainty. Corresponding covariance matrices are built for $\gamma$ and $\pi^{0}$ reconstruction, energy calibration and resolution for charged particles and $\pi^{0}$ 's, tracking and secondary interactions, and the unfolding procedure. Spectral functions are thus obtained for the leading channels: $\pi \pi^{0}, \pi 2 \pi^{0}, \pi 3 \pi^{0}, \pi 4 \pi^{0}, 3 \pi$, $3 \pi \pi^{0}, 3 \pi 2 \pi^{0}$, and $5 \pi$.

The spectral function for the $\pi \pi^{0}$ channel is given in Fig. 6, while Fig. 7 shows the consistency between the unfolded mass spectra in the $3 \pi$ and $\pi 2 \pi^{0}$ channels.

The inclusive vector $(V)$ and axial-vector $(A)$ spectral functions are determined (Fig. 8). They are respectively dominated by the low-lying $\rho$ and $a_{1}$ resonances and are shown to approach the smooth QCD prediction at larger masses, although they are clearly not 'asymptotic' at the $\tau$ mass. The convergence to the perturbative QCD regime is much better realized for the $V+A$ spectral function, as shown in Fig. 9, while the $V-A$ part in Fig. 10 undergoes large damped oscillations around zero.

The QCD analysis of the final spectral functions is in progress.

\section{Conclusions}

The final analysis of $\tau$ decay branching fractions using all LEP-I data with the ALEPH detector is presented. As in the publication based on the 1991-1993 data it uses a global analysis of all modes, classified according to charged particle identification, and charged particle and $\pi^{0}$ 
Table 4

A summary list of ALEPH branching ratios (\%). The labels $V, A$ and $S$ refer to the nonstrange vector and axialvector, and strange components, respectively. The $\omega$ decay modes marked $(*)$ are electromagnetic $\left(\pi^{0} \gamma, \pi^{+} \pi^{-}\right)$.

\begin{tabular}{|c|c|c|}
\hline mode & $\mathrm{B} \pm \sigma_{\text {tot }}[\%]$ & ALEPH prelim. \\
\hline $\bar{e}$ & $17.837 \pm 0.080$ & \\
\hline$\mu$ & $17.319 \pm 0.077$ & \\
\hline$\pi^{-}$ & $10.828 \pm 0.105$ & $\overline{\mathrm{A}}$ \\
\hline$\pi^{-} \pi^{0}$ & $25.471 \pm 0.129$ & V \\
\hline$\pi^{-} 2 \pi^{0}$ & $9.239 \pm 0.124$ & $\mathrm{~A}$ \\
\hline$\pi^{-} 3 \pi^{0}$ & $0.977 \pm 0.090$ & V \\
\hline$\pi^{-} 4 \pi^{0}$ & $0.112 \pm 0.051$ & $\mathrm{~A}$ \\
\hline$\pi^{-} \pi^{-} \pi^{+}$ & $9.041 \pm 0.097$ & A \\
\hline$\pi^{-} \pi^{-} \pi^{+} \pi^{0}$ & $4.590 \pm 0.086$ & V \\
\hline$\pi^{-} \pi^{-} \pi^{+} 2 \pi^{0}$ & $0.392 \pm 0.046$ & $\mathrm{~A}$ \\
\hline$\pi^{-} \pi^{-} \pi^{+} 3 \pi^{0}$ & $0.013 \pm 0.010$ & V \\
\hline $3 \pi^{-} 2 \pi^{+}$ & $0.072 \pm 0.015$ & A \\
\hline $3 \pi^{-} 2 \pi^{+} \pi^{0}$ & $0.014 \pm 0.009$ & $\mathrm{~V}$ \\
\hline$\pi^{-} \pi^{0} \eta$ & $0.180 \pm 0.045$ & V \\
\hline$(3 \pi)^{-} \eta$ & $0.039 \pm 0.007$ & A \\
\hline$a_{1}^{-}\left(\rightarrow \pi^{-} \gamma\right)$ & $0.040 \pm 0.020$ & A \\
\hline$\pi^{-} \omega(*)$ & $0.253 \pm 0.018$ & V \\
\hline$\pi^{-} \pi^{0} \omega(*)$ & $0.048 \pm 0.009$ & $\mathrm{~A}$ \\
\hline$(3 \pi)^{-} \omega(*)$ & $0.003 \pm 0.003$ & $\mathrm{~V}$ \\
\hline$K^{-} K^{0}$ & $0.163 \pm 0.027$ & $\mathrm{~V}$ \\
\hline$K^{-} \pi^{0} K^{0}$ & $0.145 \pm 0.027$ & $(75 \pm 25) \% \mathrm{~A}$ \\
\hline$\pi^{-} K^{0} \overline{K^{0}}$ & $0.153 \pm 0.035$ & $(75 \pm 25) \% \mathrm{~A}$ \\
\hline$K^{-} K^{+} \pi^{-}$ & $0.163 \pm 0.027$ & $(75 \pm 25) \% \mathrm{~A}$ \\
\hline$(K \bar{K} \pi \pi)^{-}$ & $0.05 \pm 0.02$ & $(50 \pm 50) \% \mathrm{~A}$ \\
\hline$K^{-}$ & $0.696 \pm 0.029$ & $\mathrm{~S}$ \\
\hline$K^{-} \pi^{0}$ & $0.444 \pm 0.035$ & S \\
\hline$\overline{K^{0}} \pi^{-}$ & $0.917 \pm 0.052$ & S \\
\hline$K^{-} 2 \pi^{0}$ & $0.056 \pm 0.025$ & S \\
\hline$K^{-} \pi^{+} \pi^{-}$ & $0.214 \pm 0.047$ & S \\
\hline$\overline{K^{0}} \pi^{-} \pi^{0}$ & $0.327 \pm 0.051$ & S \\
\hline$(K 3 \pi)^{-}$ & $0.076 \pm 0.044$ & $\mathrm{~S}$ \\
\hline$K^{-} \eta$ & $0.029 \pm 0.014$ & $\mathrm{~S}$ \\
\hline
\end{tabular}

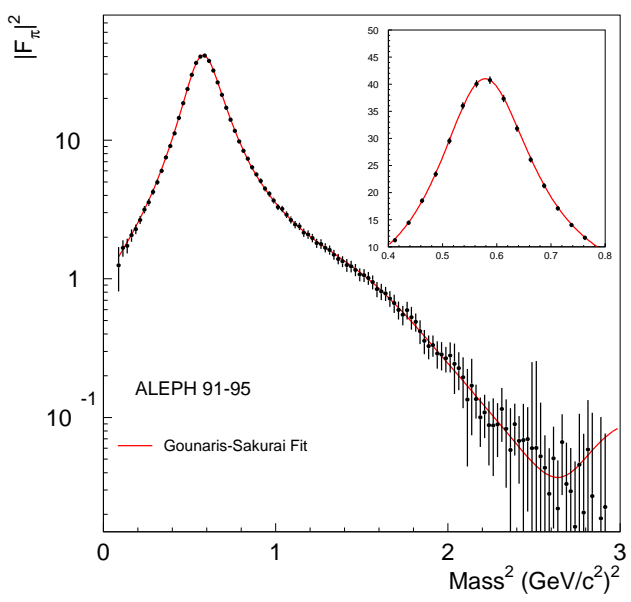

Figure 6. The $\pi^{-} \pi^{0}$ spectral function fitted using the Gounaris-Sakurai parametrization with contibutions from the $\rho, \rho$ ', and $\rho "$ resonances.

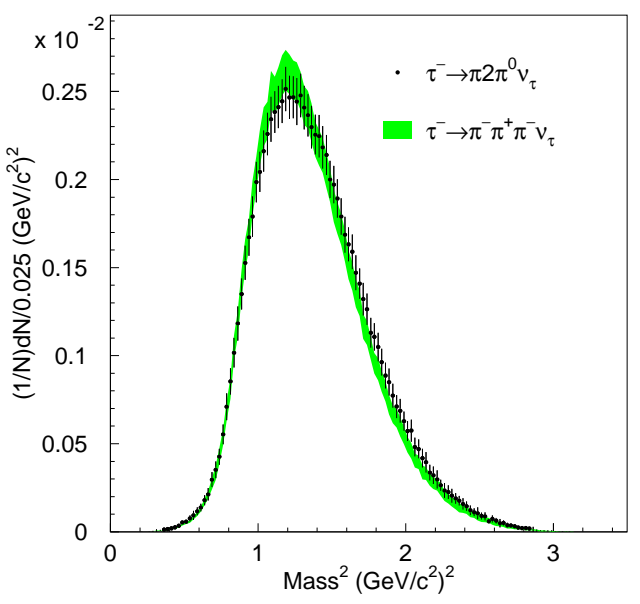

Figure 7. Comparison of the corrected mass spectra for the $3 \pi$ and $\pi 2 \pi^{0}$ hadronic modes. 

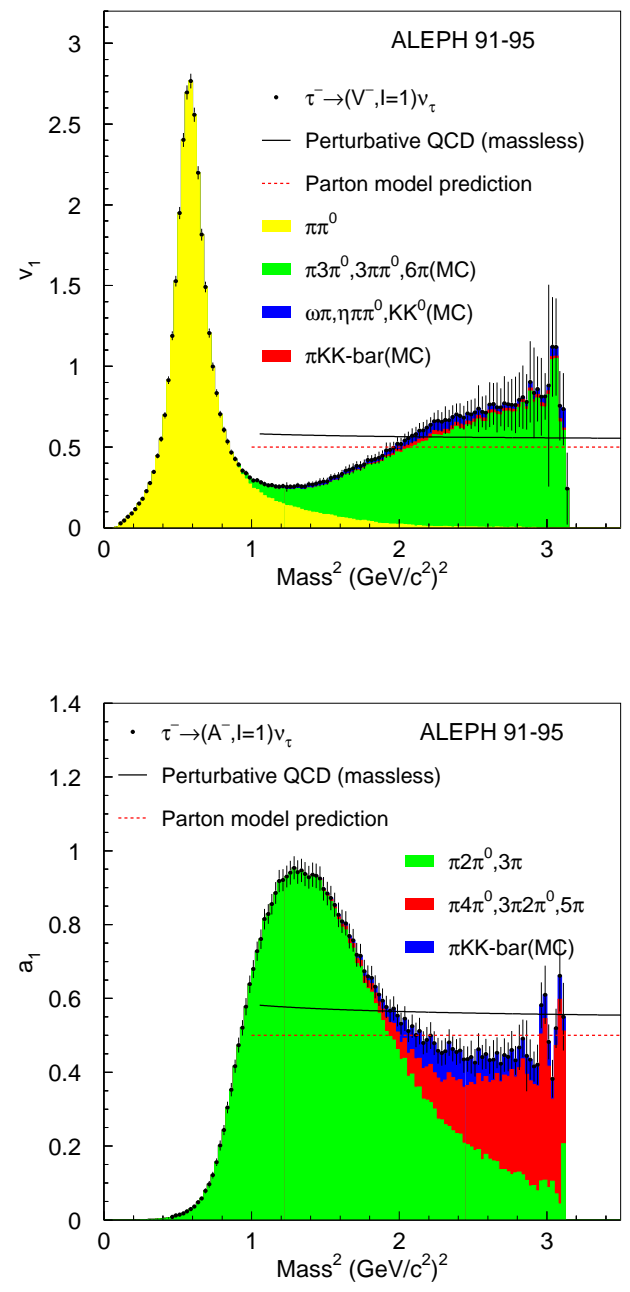

Figure 8. The vector and axial-vector spectral functions, with contributions from exclusive channels indicated (shapes of contributions labeled "MC" taken from the simulation). The QCD prediction is obtained with $\alpha_{s}\left(M_{\mathrm{Z}}^{2}\right)=$ 0.120 .

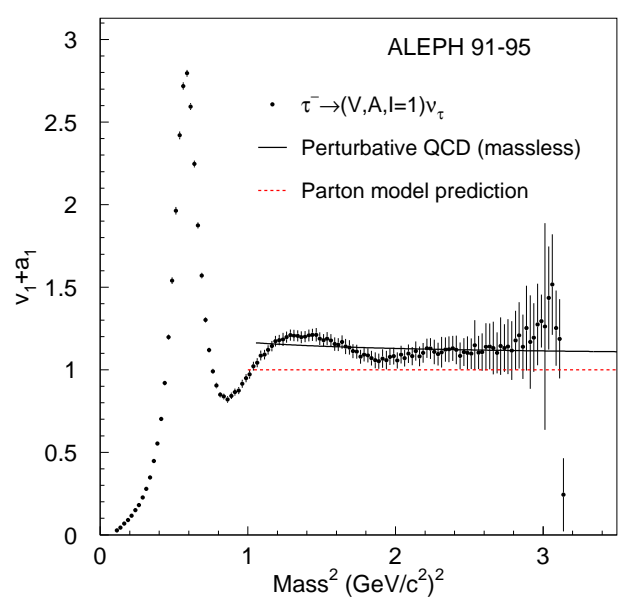

Figure 9. The inclusive $V+A$ spectral function and predictions from the parton model and from massless perturbative QCD using $\alpha_{s}\left(M_{\mathrm{Z}}^{2}\right)=$ 0.120 .

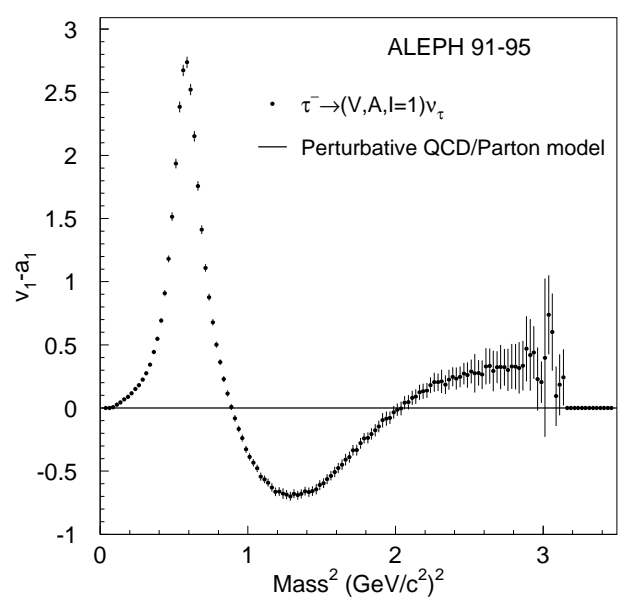

Figure 10. The $V-A$ spectral function. In the parton model as well as in perturbative QCD vector and axial-vector contributions are degenerate. 
multiplicity up to $4 \pi^{0}$ 's in the final state. Major improvements are introduced with respect to the published analysis and a better understanding is achieved, in particular in the separation between genuine and fake photons. As modes with kaons $\left(K^{ \pm}, K_{S}^{0}\right.$, and $\left.K_{L}^{0}\right)$ have already been studied and published with the full statistics, the nonstrange modes without kaons are emphasized. Taken together these results provide a complete description of the $\tau$ decay modes up to 6 hadrons in the final state.

The measured branching ratio values are internally consistent and agree with known constraints from other measurements in the framework of the Standard Model. The precision reached and the completeness of the results are for the moment unique. More specifically, the results on the leptonic and pionic fractions lead to powerful tests of universality in the charged leptonic weak current, showing that the $e-\mu-\tau$ couplings are equal within 2-3 per mille. The branching ratio of $\tau \rightarrow \nu_{\tau} \pi \pi^{0}$, which is of particular interest to the accurate determination of vacuum polarization effects, is determined with a precision of $0.5 \%$ to be $(25.47 \pm 0.13) \%$. Also the ratio of $a_{1}$ branching fractions into $\pi 2 \pi^{0}$ and $3 \pi$ final states is measured to be $0.979 \pm 0.018$, in agreement with expectation from partial wave analyses of these decays. Separating nonstrange hadronic channels into vector $(\mathrm{V})$ and axial-vector (A) components and normalizing to the electronic width yields the ratios $R_{\tau, V}=1.787 \pm 0.013$, $R_{\tau, A}=1.695 \pm 0.013, R_{\tau, V+A}=3.482 \pm 0.014$ and $R_{\tau, V-A}=0.092 \pm 0.023$.

The spectral functions for the main hadronic modes have been extracted from the mass distributions. The separated vector and axial-vector components are the basic input for QCD analyses and vacuum polarization calculations.

\section{Acknowledgements}

I would like to thank the organizers of the Tau04 Workshop for their hospitality and their efficient running of the meeting. Many thanks are due to my colleagues Changzheng Yuan and Zhiqing Zhang for their major contributions to this analysis.

\section{REFERENCES}

1. ALEPH Coll., Z. Phys. C70 (1996) 561.

2. ALEPH Coll., Z. Phys. C70 (1996) 579.

3. ALEPH Coll., Z. Phys. C54 (1992) 211.

4. ALEPH Coll., Phys. Lett. B332 (1994) 209.

5. ALEPH Coll., Phys. Lett. B332 (1994) 219.

6. ALEPH Coll., Eur. Phys. J. C1 (1998) 65.

7. ALEPH Coll., Eur. Phys. J. C4 (1998) 29.

8. ALEPH Coll., Eur. Phys. J. C10 (1999) 1.

9. ALEPH Coll., Eur. Phys. J. C11 (1999) 599.

10. ALEPH Coll., Nucl. Instr. Methods A294 (1990) 127.

11. ALEPH Coll., Nucl. Instr. Methods, A360 (1995) 481.

12. S. Jadach, B.F.L. Ward, and Z. Was, Comp. Phys. Comm. 79 (1994) 503.

13. S. Jadach et al., Comp. Phys. Comm. 76 (1993) 361.

14. ALEPH Coll., Z. Phys. C62 (1994) 539.

15. ALEPH Coll., Eur. Phys. J. C20 (2001) 401.

16. E. Barberio, B. van Eijk and Z. Was, Comp. Phys. Comm.66 (1991) 115;E. Barberio and Z. Was, Comp. Phys. Comm.79 (1994) 291.

17. S. Snow, Proceedings of the $2^{\text {nd }}$ International Workshop on $\tau$ Lepton Physics, Colombus 1992, K. K. Gan ed., World Scientific (1993).

18. ALEPH Coll., Z. Phys. C74 (1997) 263.

19. Review of Particle Physics, K.Hagiwara et al., Phys. Rev. D66 (2002) 010001.

20. D. Bortoletto et al., CLEO Coll., Phys. Rev. Lett. 71 (1993) 1791.

21. T. Bergfeld et al., CLEO Coll., Phys. Rev. Lett. 79 (1997) 2406; A. Weinstein, Proceedings of the $6^{\text {th }}$ International Workshop on $\tau$ Lepton Physics, Victoria 2000, R. J. Sobie and J. M. Roney eds., North Holland (2001).

22. M. Zielinski et al., Phys. Rev. Lett. 52 (1984) 1195.

23. A. Anastassov et al., CLEO Coll., Phys. Rev. Lett. 86 (2001) 4467.

24. ALEPH Coll., Proceedings of the Rencontre de Moriond (1999).

25. W. Marciano and A. Sirlin, Phys. Rev. Lett. 61 (1988) 1815.

26. D.I. Britton et al., Phys. Rev. Lett. 68 (1992) 3000.

27. C. Czapek et al., Phys. Rev. Lett. 70 (1993) 
17.

28. J. Z. Bai et al., Phys. Rev. D53 (1996) 20.

29. R. Decker and M. Finkemeier, Phys. Rev. D48 (1993) 4203.

30. D. Asner et al., CLEO Coll., Phys. Rev. D61 (2000) 012002.

31. R. Alemany, M. Davier and A. Höcker, Eur. Phys. J. C2 (1998) 123.

32. M. Davier and A. Höcker, Phys. Lett. B419 (1998) 419.

33. M. Davier and A. Höcker, Phys. Lett. B435 (1998) 427.

34. M. Davier, S. Eidelman, A. Höcker and Z. Zhang, Eur. Phys. J. C27 (2003) 497.

35. S. Anderson et al., CLEO Coll., Phys. Rev. D61 (2000) 112002.

36. R.R. Akhmetshin et al., CMD-2 Coll., Phys. Lett. B527 (2002) 161.

37. R.R. Akhmetshin et al., CMD-2 Coll., Phys. Lett. B578 (2004) 285.

38. M. Davier, S. Eidelman, A. Höcker and Z. Zhang, Eur. Phys. J. C31 (2003) 503.

39. G. W. Bennett, et al., Muon $(g-2)$ Coll., Phys. Rev. Lett. 92 (2004) 161802.

40. M. Bishai et al., CLEO Coll., Phys. Rev. Lett. 82 (1999) 281.

41. A. Höcker and V. Kartvelishvili, Nucl. Inst. Meth. A372 (1996) 469. 